\title{
A GEOMETRIC LOWER BOUND ON GRAD'S NUMBER
}

\author{
Alessio Figalli ${ }^{1}$
}

\begin{abstract}
In this note we provide a new geometric lower bound on the so-called Grad's number of a domain $\Omega$ in terms of how far $\Omega$ is from being axisymmetric. Such an estimate is important in the study of the trend to equilibrium for the Boltzmann equation for dilute gases.
\end{abstract}

Mathematics Subject Classification. 49Q20, 49J40.

Received January 13, 2008.

Published online April 26, 2008.

\section{INTRODUCTION AND STATEMENT OF THE RESULT}

In a recent paper [3], Desvillettes and Villani proved a rate of convergence to equilibrium for solutions of the Boltzmann equation like $O\left(t^{-\infty}\right)$ under some suitable assumptions on the solution and on the domain. In particular, the shape of the domain plays a crucial role in the uniqueness of the steady state. Indeed, for a generic shape of the domain a steady state would be a rest state (that is the density and temperature would be constant all over the box, and there would be no macroscopic velocity field), while, if the domain presents an axis of symmetry, there are steady states which are not at rest and possess a rotating velocity field. Thus, in order to prove a result of convergence to the equilibrium with a quantitative rate of convergence, one of the steps in [3] consists in expressing how much the domain deviates from axisymmetry (see [5] for the study of convergence to equilibrium in the axisymmetric case). We recall that, for $N=2,3$, a set is said axisymmetric if it has a circular symmetry around some point $(N=2)$ of if it admits an axis of symmetry $(N=3)$ (see [2], Def.-Lem. 5, for the definition in the case $N \geq 4^{2}$ ).

The way in which the absence of an axis of symmetry enters in proof of the convergence result is trough the following Korn-type inequality [2], Theorem 3 (here and in the sequel, given a vector field $u, \nabla u=\nabla^{\mathrm{sym}} u+\nabla^{a} u$ denotes the decomposition of the matrix $\nabla u$ into its symmetric and antisymmetric part):

Theorem 1.1. Let $\Omega$ be a $C^{1}$ bounded, non-axisymmetric open subset of $\mathbb{R}^{N}$, with $N \geq 2$. Let $u$ be a vector field on $\Omega$ with $\nabla u \in L^{2}(\Omega)$. Assume that $u$ is tangent to $\partial \Omega$ :

$$
u(x) \cdot n(x)=0 \quad \forall x \in \partial \Omega,
$$

Keywords and phrases. Grad's number, Korn-type inequality, axisymmetry of the domain, trend to equilibrium for the Boltzmann equation.

1 Université de Nice-Sophia Antipolis, Laboratoire J.-A. Dieudonné, UMR 6621, Parc Valrose, 06108 Nice Cedex 02, France; figalli@unice.fr

${ }^{2}$ In this paper we consider only the cases $N=2,3$, which are the most interesting from a physical point of view. However it could be possible that our techniques can be employed also in the case $N \geq 4$. 
where $n(x)$ stands for the outer unit normal vector to $\Omega$ at the point $x$. Then there exists a constant $K(\Omega)>0$, depending only on $\Omega$, such that

$$
\left\|\nabla^{\mathrm{sym}} u\right\|_{L^{2}(\Omega)}^{2} \geq K(\Omega)\|\nabla u\|_{L^{2}(\Omega)}^{2} .
$$

The value of the constant $K(\Omega)$ appearing in the above proposition is used in the study of trend to equilibrium to quantify the deviation of $\Omega$ from axisymmetry. It is therefore of great interest to have as much insight as possible in the explicit value of $K(\Omega)$, in terms of the geometry of $\Omega$. As proved in [2], Theorem 3, a lower bound on $K(\Omega)$ can be given in terms of other constants depending only on the domain:

$$
\frac{1}{K(\Omega)} \leq 2 N\left(1+C_{H}(\Omega)\right)\left(1+\frac{1}{\bar{K}(\Omega)}\right)\left(1+\frac{1}{G(\Omega)}\right) .
$$

Here $C_{H}(\Omega)$ is a constant related to the homology of $\Omega$ and the Hodge decomposition (for instance $C_{H}(\Omega)=1$ if $\Omega$ is convex), $\bar{K}(\Omega)$ is related to Korn's inequality, and $G(\Omega)$ is what the authors call the Grad's number:

$$
G(\Omega):=\frac{1}{2|\Omega|} \inf _{\Sigma \in U A_{N}} \inf _{v \in V_{\Sigma}}\left\|\nabla^{\mathrm{sym}} v\right\|_{L^{2}(\Omega)}^{2},
$$

where $U A_{N}$ denotes the set of antisymmetric $N \times N$ matrices with unit norm $\left(\Sigma=\left(\Sigma_{i j}\right) \in U A_{N}\right.$ if $\Sigma_{i j}=-\Sigma_{j i}$ and $\left.\sum_{i j}\left(\Sigma_{i j}\right)^{2}=1\right)$, and $V_{\Sigma} \subset H^{1}(\Omega)$ is the set of vector fields satisfying

$$
\left\{\begin{array}{cc}
\nabla \cdot v=0, & \nabla^{a} v=\Sigma \quad \text { in } \Omega \\
v \cdot n=0 & \text { on } \partial \Omega .
\end{array}\right.
$$

As explained in [2], Section 2, all the relevant information about axisymmetry lies in $G(\Omega)$. Thus what is important is to give quantitative estimates on the positivity of $G(\Omega)$ in terms of some geometric informations about how far $\Omega$ is from being axisymmetric.

In order to state our result, we first give two definitions. We will denote by $\mathscr{H}^{N-1}$ the $(N-1)$-dimensional Hausdorff measure.

Definition 1.2 (trace constant). Let $\Omega \subset \mathbb{R}^{N}$ be a $C^{1}$ bounded open domain. We define its trace constant $T(\Omega)$ as

$$
\frac{1}{T(\Omega)}:=\inf \left\{\frac{\mathscr{H}^{N-1}(\partial E \cap \Omega)}{\mathscr{H}^{N-1}(\partial E \cap \partial \Omega)}: E \subset \Omega,|E| \leq \frac{|\Omega|}{2}, \partial E \text { is } C^{1}\right\} .
$$

By the coarea formula for smooth function (see [1], Th. 3.40, or [6], Th. 5.4.4, for a more general result), it is not difficult to prove that

$$
\int_{\Omega}|\nabla f| \geq \frac{1}{T(\Omega)} \inf _{c \in \mathbb{R}} \int_{\partial \Omega}|f-c| \mathrm{d} \mathscr{H}^{N-1} \quad \forall f \in C^{\infty}\left(\mathbb{R}^{n}\right)
$$

(see for example [4], Lem. 3.1). We remark that one could have introduced $T(\Omega)$ directly as the smallest constant for which (1.1) holds. However, we preferred to introduce it in this other way because we believe that this presentation clarifies the geometric meaning of $T(\Omega)$.

We now introduce the notion of quadratic oscillation. Recall that, given a set $\Gamma$ and a function $f: \Gamma \rightarrow \mathbb{R}$, $\operatorname{osc}_{\Gamma} f:=\sup _{\Gamma} f-\inf _{\Gamma} f$.

Definition 1.3 (quadratic oscillation). Let $\Omega \subset \mathbb{R}^{2}$ be a $C^{1}$ bounded open domain, and decompose its boundary as a union of $C^{1}$ closed curves, that is $\partial \Omega=\cup_{i} \Gamma_{i}$. Then we define the quadratic oscillation of $\partial \Omega$ as

$$
\operatorname{osc}_{2}(\partial \Omega):=\sum_{i} \operatorname{osc}_{\Gamma_{i}}|x|^{2}
$$


Our result is the following:

Theorem 1.4. Let $\Omega \subset \mathbb{R}^{N}$ be a $C^{1}$ bounded open domain.

- If $N=2$, then

$$
G(\Omega) \geq \frac{1}{32|\Omega|^{2} T(\Omega)^{2}} \inf _{x_{0} \in \mathbb{R}^{2}} \operatorname{osc}_{2}\left(x_{0}+\partial \Omega\right)^{2} .
$$

If moreover we assume $\partial \Omega$ connected, we have

$$
G(\Omega) \geq \frac{1}{2 \mathscr{H}^{1}(\partial \Omega)^{2} T(\Omega)^{2}} \inf _{x_{0} \in \mathbb{R}^{2}} d_{H}\left(x_{0}+\partial \Omega, S_{r(\partial \Omega)}\right)^{2},
$$

where $d_{H}\left(x_{0}+\partial \Omega, S_{r(\partial \Omega)}\right)$ denotes the Hausdorff distance between $x_{0}+\partial \Omega$ and the circle $S_{r(\partial \Omega)}$ centered at the origin and with radius $r(\partial \Omega):=\frac{\sup _{\partial \Omega}|x|+\inf _{\partial \Omega}|x|}{2}$.

- If $N=3$, for $\omega \in \mathbb{R}^{3}$ we define $\partial \Omega_{\omega, t}:=\partial \Omega \cap\{\omega \cdot x=t\}$. Then, looking at $x_{0}+\partial \Omega_{\omega, t}$ as a subset of $\left(x_{0}+\{\omega \cdot x=t\}\right) \simeq \mathbb{R}^{2}$, we have

$$
G(\Omega) \geq \frac{1}{48|\Omega|^{2} T(\Omega)^{2}} \inf _{x_{0}, \omega \in \mathbb{R}^{3}}\left(\int_{\mathbb{R}} \operatorname{osc}_{2}\left(x_{0}+\partial \Omega_{\omega, t}\right) \mathrm{d} t\right)^{2}
$$

If moreover $\Omega$ is convex (so that $\partial \Omega_{\omega, t}$ is connected for almost all $t$ ), we get

$$
G(\Omega) \geq \frac{1}{3|\Omega|^{2} T(\Omega)^{2}} \inf _{x_{0}, \omega \in \mathbb{R}^{3}}\left(\int_{\mathbb{R}} \frac{\mathscr{H}^{2}\left(\Omega_{\omega, t}\right)}{\mathscr{H}^{1}\left(\partial \Omega_{\omega, t}\right)} d_{H}\left(x_{0}+\partial \Omega_{\omega, t}, S_{r\left(\partial \Omega_{\omega, t}\right)}\right) \mathrm{d} t\right)^{2} .
$$

Remark 1.5 (the case $N=2$ ). By the above theorem we know that Grad's number can be bounded from below by the sum of the oscillations (in the $L^{\infty}$ norm) of $|x|^{2}$ on each connected component of $\partial \Omega$ (once $\Omega$ has been properly translated). This means that, if $G(\Omega)$ is small, each connected component of $\partial \Omega$ must be contained in some annulus $\left\{c_{1} \leq|x| \leq c_{2}\right\}$, with $c_{2}-c_{1}$ small. However, even if we assume $\Omega$ to be connected and simply connected, this does not imply that $\Omega$ is close to a disc, since it could be entirely contained in an annulus.

On the other hand, if we assume $\Omega$ to be convex, it is simple to see that $G(\Omega)$ controls in a quantitative way the Hausdorff distance between $\Omega$ and a disc. We also remark that in this case the constant $C_{H}(\Omega)$ appearing in [2], Theorem 3, can be taken equal to 1 (see [2], Appendix), and so our estimate gives a good lower bound for the constant $K(\Omega)$ appearing in Theorem 1.1.

Remark 1.6 (the case $N=3$ ). The geometric quantity that we control in this case, although more complicated than in dimension two, is quite natural: for instance, if a set $\Omega$ is "almost axisymmetric" with respect to an axis $\omega$, we expect that for almost all $t \in \mathbb{R}$ the set $\Omega \cap\{\omega \cdot x=t\}$ will be "almost radially symmetric". On the other hand, since some level sets can be close to balls while others can be close to annuli, the behavior of $\Omega$ in the direction $\omega$ can be arbitrary.

Let us also observe that the vector $x_{0}$ appearing both in (1.4) and (1.5) corresponds just to a translation of the whole set $\Omega$. Therefore the sets $\Omega_{\omega, t}$ are translated by the same quantity for all $t \in \mathbb{R}$.

Remark 1.7. Our lower bound on $G(\Omega)$ is in general stronger than the one in [2]. For example, let $\Omega$ be either a smoothed version of a ball in $\mathbb{R}^{2}$ from which an $\varepsilon$-strip has been removed, say

$$
\Omega \simeq\left\{\left(x_{1}, x_{2}\right) \in \mathbb{R}^{2} \mid x_{1}^{2}+x_{2}^{2} \leq 1\right\} \backslash\left\{\left(x_{1}, x_{2}\right) \in \mathbb{R}^{2}\left|0 \leq x_{1} \leq 1,\right| x_{2} \mid \leq \varepsilon\right\}
$$

or a smoothed version of an annulus from which an $\varepsilon$-strip has been removed, say

$$
\Omega \simeq\left\{\left(x_{1}, x_{2}\right) \in \mathbb{R}^{2} \mid 1 / 4 \leq x_{1}^{2}+x_{2}^{2} \leq 1\right\} \backslash\left\{\left(x_{1}, x_{2}\right) \in \mathbb{R}^{2}\left|0 \leq x_{1} \leq 1,\right| x_{2} \mid \leq \varepsilon\right\} .
$$


Then in both cases $T(\Omega) \sim 1$, and from (1.3) we get $G(\Omega) \geq c$, while the estimate provided by [2], Equation (26), is $G(\Omega) \geq c \varepsilon^{2}$.

Another advantage of our lower bound is its simplicity for practical computation. For example, if $\Omega$ is a slightly elongated ellipse in the plane, say

$$
\Omega=\left\{\left(x_{1}, x_{2}\right) \in \mathbb{R}^{2} \mid x_{1}^{2}+\frac{x_{2}^{2}}{(1+\varepsilon)} \leq 1\right\}
$$

then (1.3) immediately implies $G(\Omega) \geq c \varepsilon^{2}$, and we recover the estimate proved at the end of Section 4 in [2].

\section{Proof of Theorem 1.4}

Let $v \in V_{\Sigma}$ with $\Sigma \in U A_{N}$, and consider the rigid motion $R: \mathbb{R}^{n} \rightarrow \mathbb{R}^{n}$ defined by

$$
R(x):=\Sigma x .
$$

Then, by (1.1) applied to each component of the function $v-R=\left(v^{1}-R^{1}, \ldots, v^{N}-R^{N}\right)$, we get

$$
\begin{aligned}
\sqrt{|\Omega|} \sqrt{\int_{\Omega}\left|\nabla^{\mathrm{sym}} v\right|^{2} \mathrm{~d} x} & =\sqrt{|\Omega|} \sqrt{\int_{\Omega}|\nabla(v-R)|^{2} \mathrm{~d} x}=\sqrt{|\Omega|} \sqrt{\sum_{i} \int_{\Omega}\left|\nabla\left(v^{i}-R^{i}\right)\right|^{2} \mathrm{~d} x} \\
& \geq \sqrt{|\Omega|} \frac{1}{\sqrt{N}} \sum_{i} \sqrt{\int_{\Omega}\left|\nabla\left(v^{i}-R^{i}\right)\right|^{2} \mathrm{~d} x} \\
& \geq \frac{1}{\sqrt{N}} \sum_{i} \int_{\Omega}\left|\nabla\left(v^{i}-R^{i}\right)\right| \mathrm{d} x \\
& \geq \frac{1}{\sqrt{N} T(\Omega)} \sum_{i} \inf _{c^{i} \in \mathbb{R}} \int_{\partial \Omega}\left|v^{i}-R^{i}-c^{i}\right| \mathrm{d} \mathscr{H}^{N-1}(x) \\
& \geq \frac{1}{\sqrt{N} T(\Omega)} \inf _{c \in \mathbb{R}^{N}} \int_{\partial \Omega}|v-\Sigma x-c| \mathrm{d} \mathscr{H}^{N-1}(x) \\
& \geq \frac{1}{\sqrt{N} T(\Omega)} \inf _{c \in \mathbb{R}^{N}} \int_{\partial \Omega}|(v-\Sigma x-c) \cdot n| \mathrm{d} \mathscr{H}^{N-1}(x) \\
& =\frac{1}{\sqrt{N} T(\Omega)} \inf _{c \in \mathbb{R}^{N}} \int_{\partial \Omega}|(\Sigma x-c) \cdot n| \mathrm{d} \mathscr{H}^{N-1}(x)
\end{aligned}
$$

where we used $v \cdot n=0$ on $\partial \Omega$. We now remark that, up to a translation of $\Omega$, we can assume that the infimum in the last quantity is attained for $c=0$. Thus

$$
\sqrt{|\Omega|} \sqrt{\int_{\Omega} \mid \nabla^{\left.\operatorname{sym}_{v}\right|^{2}} \mathrm{~d} x} \geq \frac{1}{\sqrt{N} T(\Omega)} \int_{\partial \Omega}|(\Sigma x) \cdot n| \mathrm{d} \mathscr{H}^{N-1}(x)
$$

and we want to use the quantity appearing in the right hand side to control how far $\Omega$ is from being axisymmetric.

- The case $N=2$

We remark that in this case there are only two possibilities for $\Sigma$ :

$$
\Sigma= \pm\left(\begin{array}{ll}
0 & 1 / \sqrt{2} \\
-1 / \sqrt{2} & 0
\end{array}\right) .
$$


This implies that

$$
|(\Sigma x) \cdot n|=|x \cdot(\Sigma n)|=\frac{1}{\sqrt{2}}\left|x \cdot n^{\perp}\right| .
$$

Let us fix a connected component $\Gamma$ of $\partial \Omega$, and parameterize it with a curve $\gamma:[0, L] \rightarrow \mathbb{R}^{2}$, where $L=\mathscr{H}^{1}(\Gamma)$ and $\gamma$ is parameterized by arc-length. We observe that

$$
n^{\perp}(\gamma)= \pm \dot{\gamma}
$$

and so

$$
\left|\gamma \cdot n^{\perp}(\gamma)\right|=|\gamma \cdot \dot{\gamma}|
$$

Therefore

$$
\int_{\Gamma}|(\Sigma x) \cdot n| \mathrm{d} \mathscr{H}^{1}(x)=\frac{1}{\sqrt{2}} \int_{\gamma}|\gamma \cdot \dot{\gamma}| \mathrm{d} \mathscr{H}^{1}=\frac{1}{\sqrt{2}} \int_{0}^{L}|\gamma(t) \cdot \dot{\gamma}(t)| \mathrm{d} t=\left.\frac{1}{2 \sqrt{2}} \int_{0}^{L}\left|\frac{\mathrm{d}}{\mathrm{d} t}\right| \gamma(t)\right|^{2} \mid \mathrm{d} t .
$$

This gives

$$
\operatorname{osc}_{\Gamma}|x|^{2}:=\sup _{\Gamma}|x|^{2}-\inf _{\Gamma}|x|^{2} \leq\left.\int_{0}^{L}\left|\frac{\mathrm{d}}{\mathrm{d} t}\right| \gamma(t)\right|^{2}\left|\mathrm{~d} t=2 \sqrt{2} \int_{\Gamma}\right|(\Sigma x) \cdot n \mid \mathrm{d} \mathscr{H}^{1} .
$$

If we now decompose $\partial \Omega=\cup_{i} \Gamma_{i}$ into its connected components, we can write the above inequality for each $i$, and adding them we obtain

$$
\sum_{i} \operatorname{osc}_{\Gamma_{i}}|x|^{2} \leq 2 \sqrt{2} \int_{\partial \Omega}|(\Sigma x) \cdot n| \mathrm{d} \mathscr{H}^{1}(x) .
$$

Thus, recalling Definition 1.3 and using (2.1), we get

$$
\operatorname{osc}_{2}(\partial \Omega) \leq 2 \sqrt{2} \int_{\partial \Omega}|(\Sigma x) \cdot n| \mathrm{d} \mathscr{H}^{1}(x) \leq 4 T(\Omega) \sqrt{|\Omega|} \sqrt{\int_{\Omega}\left|\nabla^{\mathrm{sym}_{v}}\right|^{2} \mathrm{~d} x}
$$

We remark that $\operatorname{osc}_{2}(\partial \Omega)$ is not invariant by translations of $\Omega$ (recall that we assumed $\Omega$ to be translated in such a way that $\inf _{c \in \mathbb{R}^{2}} \int_{\partial \Omega}|(\Sigma x-c) \cdot n|$ is attained at $\left.c=0\right)$. Thus we take the infimum in the left hand side among all possible translations, and the infimum in the right hand side among all $v$ and $\Sigma$. In this way we conclude

$$
\inf _{x_{0} \in \mathbb{R}^{2}} \operatorname{osc}_{2}\left(x_{0}+\partial \Omega\right) \leq 4 T(\Omega) \sqrt{|\Omega|} \inf _{\Sigma, v \in V_{\Sigma}} \sqrt{\int_{\Omega}\left|\nabla^{\mathrm{sym}} v\right|^{2} \mathrm{~d} x}
$$

which is exactly (1.2).

Let us now assume that $\partial \Omega$ is connected. In this case we can give a more geometric estimate. Indeed we observe that

$$
\sup _{\partial \Omega}|x| \geq f_{\partial \Omega}|x| \mathrm{d} \mathscr{H}^{1}(x) \geq f_{\partial \Omega} x \cdot n \mathrm{~d} \mathscr{H}^{1}(x)=\frac{1}{\mathscr{H}^{1}(\partial \Omega)} \int_{\Omega} \operatorname{div}(x) \mathrm{d} x=2 \frac{|\Omega|}{\mathscr{H}^{1}(\partial \Omega)} .
$$

Thus we have

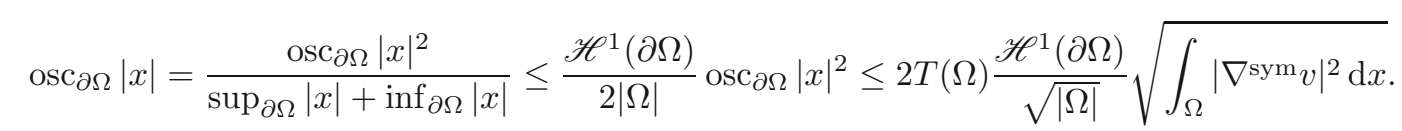

Observing that $\operatorname{osc}_{\partial \Omega}|x|$ is just twice the Hausdorff distance between $\partial \Omega$ and the circle $S_{r(\partial \Omega)}$ centered at the origin and with radius $r(\partial \Omega):=\frac{\sup _{\partial \Omega}|x|+\inf _{\partial \Omega}|x|}{2},(1.3)$ follows. 
- The case $N=3$

As we will see, we will reduce to the two-dimensional case through a coarea argument. Indeed, first we remark that to each $\Sigma$ we can associate a unit vector $\omega \in \mathbb{R}^{3}$ such that

$$
\Sigma x=\frac{1}{\sqrt{2}} \omega \wedge x .
$$

Moreover we remark that, since $\Sigma x \cdot n=-x \cdot \Sigma n$,

$$
|(\omega \wedge x) \cdot n|=|(\omega \wedge n) \cdot x| .
$$

We now use the coarea formula with respect to the map

$$
f: \partial \Omega \rightarrow \mathbb{R}, \quad f(y)=\omega \cdot y
$$

(see [1], Th. 2.93). Since the tangential Jacobian $J f(y)$ is equal to $|\omega \wedge n(y)|$ (which is just the norm of the projection of $\omega$ on the tangent space of $\partial \Omega$ at $y$ ), we have

$$
\begin{aligned}
\int_{\partial \Omega}|(\Sigma x) \cdot n| \mathrm{d} \mathscr{H}^{2}(x) & =\frac{1}{\sqrt{2}} \int_{\partial \Omega}|(\omega \wedge n) \cdot x| \mathrm{d} \mathscr{H}^{2}(x) \\
& =\frac{1}{\sqrt{2}} \int_{\partial \Omega \cap\{\omega \wedge n \neq 0\}}|(\omega \wedge n) \cdot x| \mathrm{d} \mathscr{H}^{2}(x) \\
& =\frac{1}{\sqrt{2}} \int_{\partial \Omega \cap\{\omega \wedge n \neq 0\}} \frac{|(\omega \wedge n) \cdot x|}{|\omega \wedge n|}|\omega \wedge n| \mathrm{d} \mathscr{H}^{2}(x) \\
& =\frac{1}{\sqrt{2}} \int_{\mathbb{R}} \mathrm{d} t \int_{\partial \Omega \cap\{\omega \wedge n \neq 0\} \cap\{\omega \cdot x=t\}} \frac{|(\omega \wedge n) \cdot x|}{|\omega \wedge n|} \mathrm{d} \mathscr{H}^{1}(x) .
\end{aligned}
$$

Observe that by Sard theorem the set of critical points of $f$ has measure zero. Thus for $\mathscr{L}^{1}$-a.e. $t$ the set $\{\omega \wedge n=0\} \cap\{\omega \cdot x=t\}$ is empty, and, if we define $\Omega_{\omega, t}:=\Omega \cap\{\omega \cdot x=t\}$, the set $\partial \Omega_{\omega, t}:=\partial\left(\Omega_{\omega, t}\right)=\partial \Omega \cap\{\omega \cdot x=t\}$ is a union of smooth curves. Thus, we can write

$$
\int_{\partial \Omega}|(\Sigma x) \cdot n| \mathrm{d} \mathscr{H}^{2}(x)=\frac{1}{\sqrt{2}} \int_{\mathbb{R}} \mathrm{d} t \int_{\partial \Omega_{\omega, t}} \frac{|(\omega \wedge n) \cdot x|}{|\omega \wedge n|} \mathrm{d} \mathscr{H}^{1}(x) .
$$

Now we remark that $\omega \wedge n$ is orthogonal to $\omega$, so it belongs to the plane $\Pi:=\{\omega \cdot x=0\}$. Moreover $\omega \wedge n$ is also orthogonal to $n$. These two facts implies that we can write

$$
\frac{\omega \wedge n}{|\omega \wedge n|}= \pm n_{t}^{\perp}
$$

where $n_{t}$ is the normal to the 1-dimensional set $\Gamma_{\omega, t}:=\left\{y \in \Pi: y+t \omega \in \partial \Omega_{\omega, t}\right\}$ seen as a subset of $\Pi \simeq \mathbb{R}^{2}$. Therefore we can write each $x \in \partial \Omega_{\omega, t}$ as $x=y+t \omega$ with $y \in \Gamma_{\omega, t}$, and we have

$$
\frac{|(\omega \wedge n) \cdot x|}{|\omega \wedge n|}=\left|\frac{\omega \wedge n}{|\omega \wedge n|} \cdot x\right|=\left|n_{t}^{\perp} \cdot x\right|=\left|n_{t}^{\perp} \cdot y\right| .
$$

This gives

$$
\int_{\partial \Omega}|(\Sigma x) \cdot n| \mathrm{d} \mathscr{H}^{2}(x)=\frac{1}{\sqrt{2}} \int_{\mathbb{R}} \mathrm{d} t \int_{\Gamma_{t}}\left|n_{t}^{\perp} \cdot y\right| \mathrm{d} \mathscr{H}^{1}(y) .
$$


In this way we reduced exactly to the case $N=2$ : by (2.2) we get

$$
\int_{\Gamma_{t}}\left|n_{t}^{\perp} \cdot y\right| \mathrm{d} \mathscr{H}^{1}(y) \geq \frac{1}{2} \operatorname{osc}_{2}\left(\partial \Omega_{\omega, t}\right)
$$

where $\operatorname{osc}_{2}\left(\partial \Omega_{\omega, t}\right)$ is defined as in the two-dimensional case looking at $\partial \Omega_{\omega, t}$ as a subset of $\{\omega \cdot x=t\} \simeq \mathbb{R}^{2}$. This, together with (2.3), gives

$$
\int_{\partial \Omega}|(\Sigma x) \cdot n| \mathrm{d} \mathscr{H}^{2}(x) \geq \frac{1}{2 \sqrt{2}} \int_{\mathbb{R}} \operatorname{osc}_{2}\left(\partial \Omega_{\omega, t}\right) \mathrm{d} t .
$$

Using (2.1) and taking the infimum among all translations of $\Omega$, we obtain

$$
\sqrt{|\Omega|} \sqrt{\int_{\Omega} \mid \nabla^{\left.\operatorname{sym}_{v}\right|^{2} \mathrm{~d} x}} \geq \frac{1}{2 \sqrt{6} T(\Omega)} \inf _{x_{0} \in \mathbb{R}^{3}} \int_{\mathbb{R}} \operatorname{osc}_{2}\left(x_{0}+\partial \Omega_{\omega, t}\right) \mathrm{d} t .
$$

Taking now the infimum among all $v$ in the left hand side and among all $\omega$ in both sides, we finally obtain (1.4).

If moreover we know that $\Omega$ is convex, for all $\omega$ and for almost all $t$, the set $\partial \Omega_{\omega, t}$ will consist of a unique smooth curve. Therefore, with the same notation as in the case $N=2$,

$$
\int_{\Gamma_{t}}\left|n_{t}^{\perp} \cdot y\right| \mathrm{d} \mathscr{H}^{1}(y) \geq 2 \frac{\mathscr{H}^{2}\left(\Omega_{\omega, t}\right)}{\mathscr{H}^{1}\left(\partial \Omega_{\omega, t}\right)} d_{H}\left(x_{0}+\partial \Omega_{\omega, t}, S_{r\left(\partial \Omega_{\omega, t}\right)}\right),
$$

which combined with (2.4) gives

$$
\int_{\partial \Omega}|(\Sigma x) \cdot n| \mathrm{d} \mathscr{H}^{2}(x) \geq \sqrt{2} \int_{\mathbb{R}} \frac{\mathscr{H}^{2}\left(\Omega_{\omega, t}\right)}{\mathscr{H}^{1}\left(\partial \Omega_{\omega, t}\right)} d_{H}\left(x_{0}+\partial \Omega_{\omega, t}, S_{r\left(\partial \Omega_{\omega, t}\right)}\right) \mathrm{d} t
$$

and (1.5) follows.

Acknowledgements. I warmly thank Cédric Villani for useful discussions and a careful reading of the paper.

\section{REFERENCES}

[1] L. Ambrosio, N. Fusco and D. Pallara, Functions of bounded variation and free discontinuity problems. The Clarendon Press, Oxford University Press, New York (2000).

[2] L. Desvillettes and C. Villani, On a variant of Korn's inequality arising in statistical mechanics. ESAIM: COCV 8 (2002) 603-619.

[3] L. Desvillettes and C. Villani, On the trend to global equilibrium for spatially inhomogeneous kinetic systems: the Boltzmann equation. Invent. Math. 159 (2005) 245-316.

[4] A. Figalli, F. Maggi and A. Pratelli, A mass transportation approach to quantitative isoperimetric inequalities. Preprint (2007).

[5] C. Villani, Hypocoercivity. Memoirs Amer. Math. Soc. (to appear).

[6] W.P. Ziemer, Weakly differentiable functions. Sobolev spaces and functions of bounded variation. Graduate Texts in Mathematics 120. Springer-Verlag, New York (1989). 